\title{
In-silico Studies on Phloroglucinol and SARS CoV2MPro
}

\author{
Ritesh Kumar and Kapil Hazarika \\ Department of Pharmacology Shree Guru Gobind Singh \\ Tricentary University, Gurugram, Haryana, India \\ Corresponding author email: ritesh_fmhs@sgtuniversity.org
}

\section{ABSTRACT}

A scene of emergency has caused worldwide due to the spread of a life threatening diseases identified as Covid-19 which is caused by the Severe Acute Respiratory Syndrome Corona virus 2 (SARS CoV2).Millions of people lost their life and people are still dying. There is a need of an ideal antiviral agent that can not only stop this pandemic spread but can cure the corona virus. The Main Protease $\mathrm{M}^{\text {pro }}$ is identified as the high potential drug target. Natural products are always used from the ancient times by the ancestors to treat many diseases, they are the hope to this pandemic and may stabilize the world. Phloroglucinol, active phytochemical of Brown algae is used in many viral diseases like HIV. This phytochemical is also used to treat many diseases like Alzheimer's disease, Parkinson's disease and many more. In this study we performed in-silico studies to find out whether phloroglucinol can be a promising anti-viral to cure this pandemic.

KEY W ORDS: PHLOROGLUCINOL, PHYTOCHEMICAL, SARS COV2 MAIN PROTEASE, DOCKING, POTENTIAL DRUG.

\section{INTRODUCTION}

In December 2019, Corona Virus outbreak in Wuhan, City of China,has caused a loss to millions of life globally. On 16th May 2020, there were 4.59 Million cases reported globally positive for Corona Virus and still this disease is on loose. Today, 14th October 2020, there are 38.3 Million cases worldwide and these cases are still increasing with a rapid rate in many countries like USA, India, Brazil, etc(Worldometer (2020), H.A. Diah Handayani (2015)).

Some developed and developing countries have faced a huge loss of life. These countries include USA, India and Brazil, these are the top 3 countries that are having the highest number of corona cases. Many countries are claiming that they have vaccine against the disease but still researches are going on. Figure 1 and 2 represents cases of COVID-

Biosc Biotech Res Comm P-ISSN: 0974-6455 E-ISSN: 2321-4007

\section{crossef}

Identifiers and Pagination

Year: 2021 Vol: 14 No (7) Special Issue

Pages: 278-282

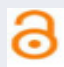

creative

Article Information

Received: $13^{\text {th }}$ May 2021

This is an open access article under Creative

Commons License Attribn 4.0 Intl (CC-BY).

DOI: $h t t p: / / d x$.doi.org/10.21786/bbrc/14.7.61
19 till 14th October and active cases in different countries respectively.

Figure 1: Covid-19 Positive Cases worldwide till 14th October 2020 (Worldometer et. al. (2020)

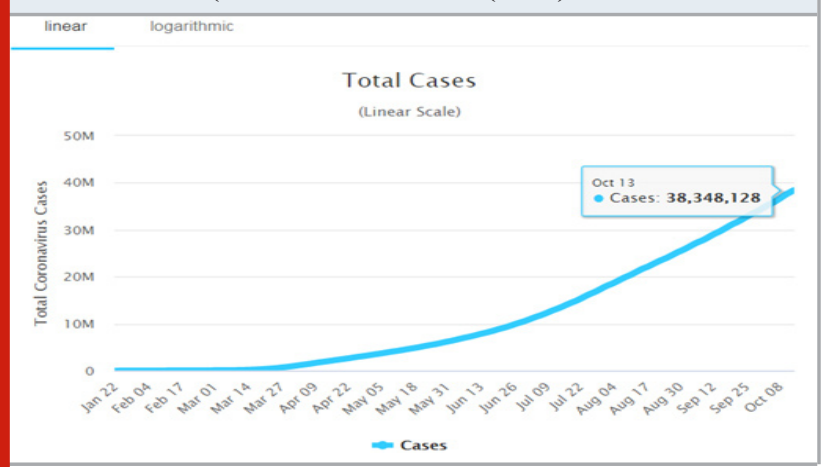

Designing an anti-corona drug is a challenge due to various variations in its amino acid sequence. The viral Main ProteaseMpro which is also called 3CLpro, is responsible for many viral replication activities. It is papain-like proteases that are responsible for the self-maturation of virus and it also helps in processing many viral replication enzymes. This protein is a potential target to develop a better $\operatorname{drug}(\mathrm{M}$. Kandeel et. al. (2020). 
Figure 2: Covid-19 Positive cases in different cases (Worldometer et. al. (2020)

Countries cases distribution

Distribution of cases

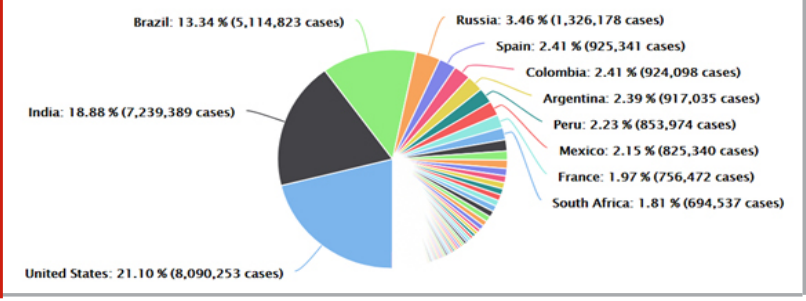

Natural Products are always in interest for the development for the better drug without any other side-effects. Natural products have been traditionally used to prevent and cure many deadly diseases. Many active ingredients of natural products are currently used by many pharmaceutical industries to prevent and cure many diseases. Active compound of Brown Algae is Phloroglucinol, it is found to be effective against many diseases. Phloroglucinol or 1,3,5trihydroxybenzene having a molecular weight of $126.11 \mathrm{~g} /$ mol with a molecular formula of $\mathrm{C}_{6} \mathrm{H}_{6} \mathrm{O}_{3}$ has been used against many diseases like Alzheimer's Disease, Parkinson's Disease, and in many other disease to reduce the oxidative stress. Phloroglucinol seems to be the natural compound than can prevent the spread of Corona Virus(S.K. Goswami et. al. (2016).

Literature Review: Chojnacka, K., et.al. reviewedabout different natural products and how their constituents can be used to inhibit or prevent the corona virus spread in human body and on Earth,one of them is Phloroglucinol. In-silico studies on compound Phloroglucinolwas performed which is a potential inhibitor SARS CoV2 Main Protease or Mpro (K. Chojnacka et. al. (2020)). Ryu, Junghwa, et al. stated the potential of phloroglucinol to cure the Parkinson'sdisease. This disease is the second most chronic disease. In this research in-vivo experiments were performed like Immunohistochemistry (IHC), western blotting, Surgeries, and many assays. Administration of phloroglucinol against the motor functional impairments was disclosed. However no discussion was made about the effects of phloroglucinol on SARS CoV2 proteins. Thus, in-silico studies were performed on Phloroglucinol which is a potential inhibitor SARS CoV2 Main Protease or Mpro(J. Ryu et. al. (2013).

Yang, Eun-Jeong, et al. performed cell viability measurements, Morris water maze test, immunohistochemistry, western blotting and other tests. Anti-oxidant properties of phloroglucinol against Alzheimer's disease was disclosed. Thus, the research represented the interaction of phloroglucinol and SARS Cov2 Main Protease. The aforementioned research may aid researchers to develop a better vaccine for corona virus(E.J. Yang et. al. (2015) Kim, Moon-Moo, and Se-Kwon Kim stated possible mechanism of inhibiting the oxidative stress and inflammation by performing various in-vivo experiments like western blotting, gelatinzymography, immunoassay and many more, however, discussion about the effects of phloroglucinol on SARS CoV2 proteins was not made. Here this research is about the in-silico studies showing how Phloroglucinol is interacting with the proteins of SARS CoV2 Main Protease(M. M. Kim et. al. (2010). Langarizadeh, Mohammad Amin, et al. with the help of in-silico studies revealed that the constituents of brown algae shows a strong anti-HIV properties.

They also showed how these phlorotannins are interrupting the HIV replication. Langarizadeh, Mohammad Amin, et al. didn't discussed about the effect of phlotannins on Sars Cov2 proteins. Here this research is about the in-silico studies on one of the compound Phloroglucinol which is a potential inhibitor SARS CoV2 Main Protease or $\mathrm{M}^{\text {pro }}(\mathrm{M}$. A. Langarizadeh et. al. (2014). Phloroglucinol have been used against many diseases like Parkinson's disease and many viral disease. However, no reports exist utilization of phloroglucinolagainst SARS CoV2 Main Protease. Current research will discuss the in-silico studies performed with Phloroglucinol on SARS CoV2 Main protease. This study will further aid the researchers and scientists to understand and carry out in-vitro studies with Phloroglucinol on SARS CoV2 Main protease.

\section{Research Questions}

1. What is the importance of Phloroglucinolto prevent spread of corona virus?

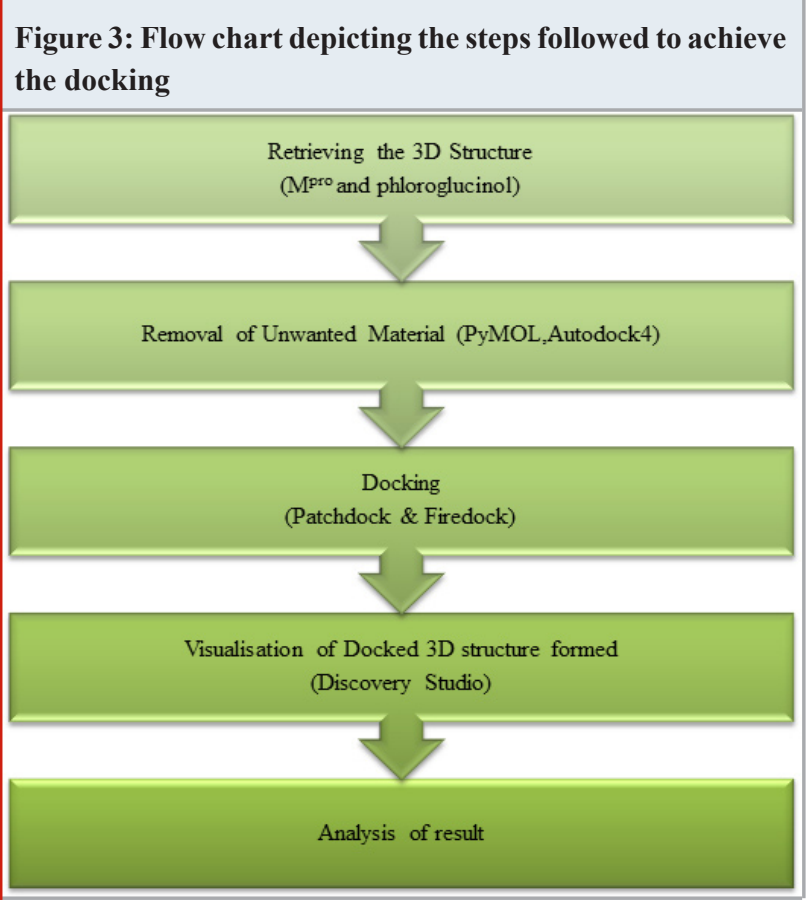

\section{METHODOLOGY}

Design: To achieve the docking a series of protocol was followed (Figure 3). Firstly, 3D conformations of Ligand that is Phloroglucinol and Protein that is $\mathrm{M}^{\mathrm{pro}}$ were downloaded from PubChem and RCSB PDB. These files have inhibitors and many unwanted molecules attached with the desired protein, these unwanted molecules were removed with the help of Autodock and PyMOL. Files in PDB format are subjected to Patchdock and Firedock. 
Results obtained in the Personal email were downloaded and visualised with the help of Drug Discovery Studio and different parameters like distance between bonds, bond type, ACE and Global energy was recorded.

Sample: Files containing the 3D structure were downloaded from RCSB PDB for protein (Figure 4) and PubChem for Ligand (Figure 5). Figure 5 represents the Quaternary structure of protein where Red spirals are depicting the $\alpha$-helices and the cyan colour ribbons are representing the $\beta$-plated sheets. In Figure 6, Ball and stick 3D structure is used to understand the placement of carbons. Green balls are depicting the carbon atoms, red balls are for oxygen and the white balls are representing the hydrogen atoms while the single line between atoms is for single bond and double line shows that there is double bond between atoms.

\section{Figure 4: Quaternary Structure of $M^{\text {pro }}$}

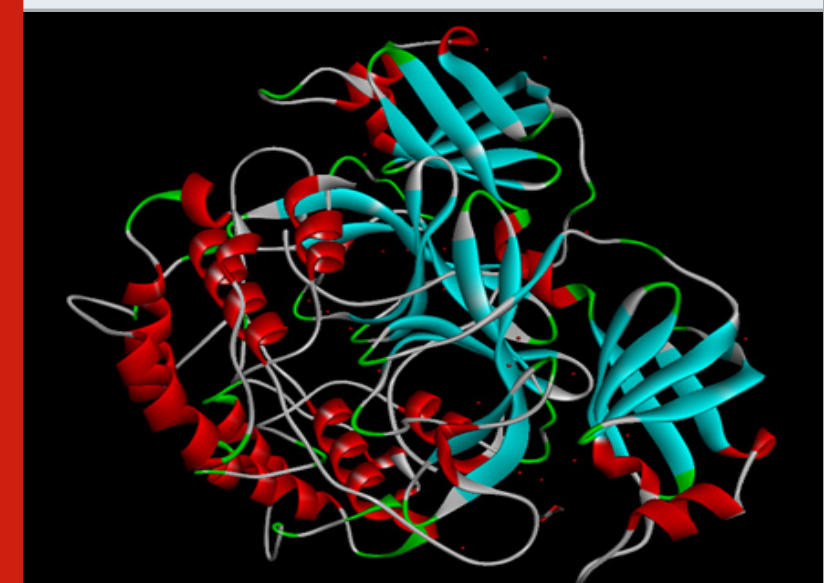

Intrument:

PubChem: 3D structure of Phloroglucinolwas downloaded from Public Chemistry (PubChem). It is the largest collection of chemical information globally which can be accessed from anywhere in the world. Information regarding the chemical properties or 3D structure of different chemical compound can be found here and in different file formats. This information is freely accessible and freely downloadable.

Figure 5: Ball and Stick Structure of Phloroglucinol

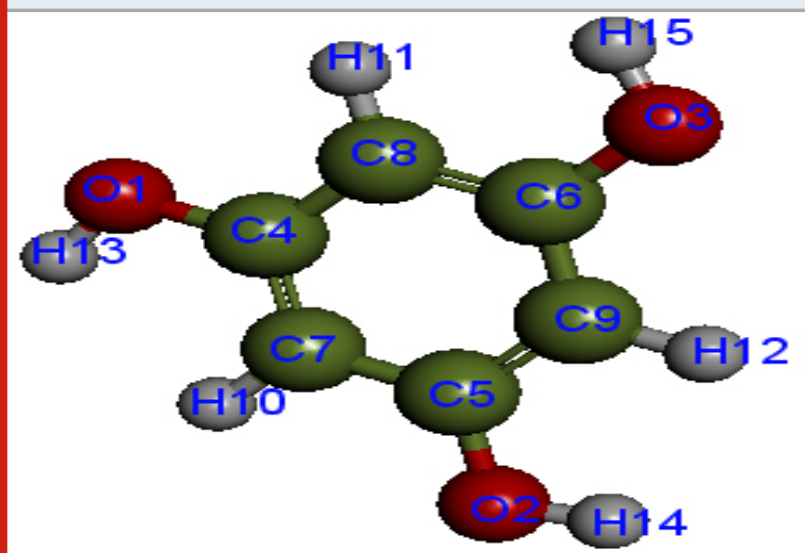

Research Collaboratory for Structural Bioinformatics (RCSB PDB): 3D structure of SARS CoV2 Main Protease was downloaded from Research Collaboratory for Structural Bioinformatics (RCSB) Protein Data Bank (PDB). Itis a database to access the three dimensional structures of biological molecules that are large in size like proteins, nucleic acids etc. This information is freely available and can be downloaded from anywhere in the world.

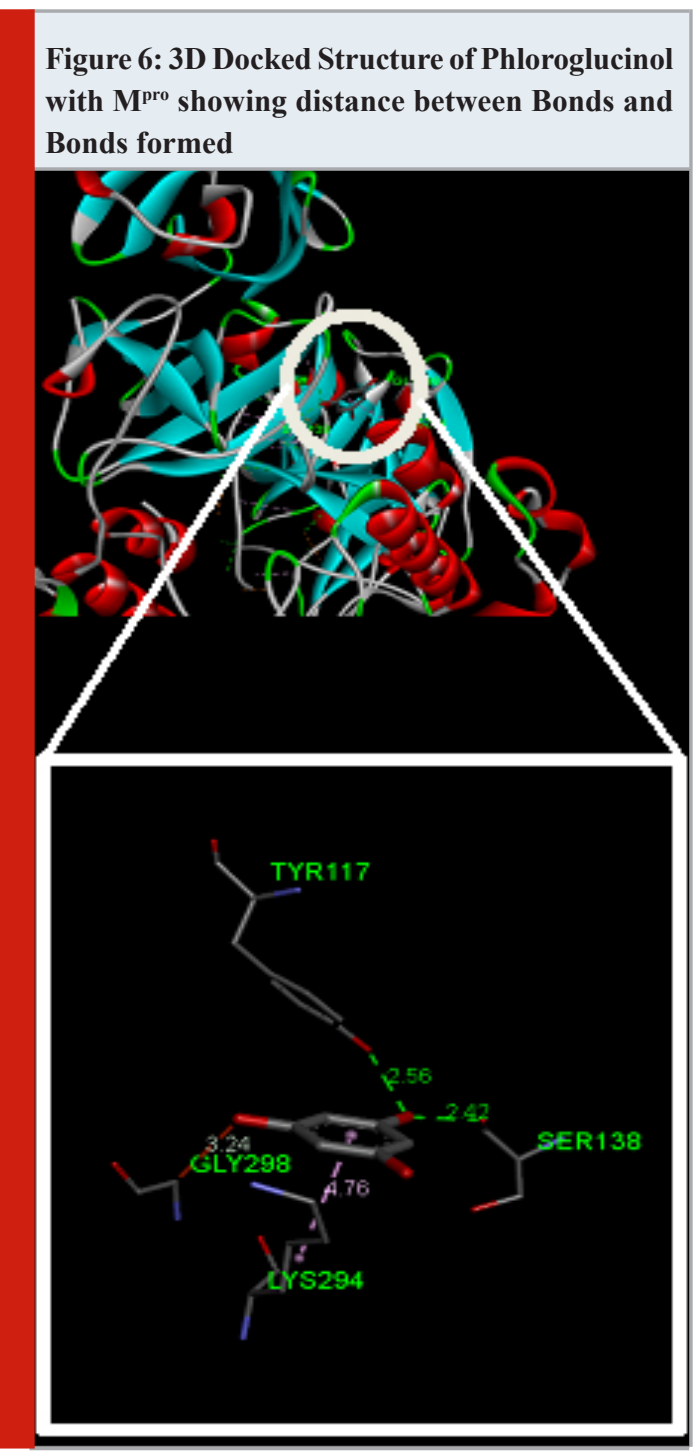

Autodock: Additional Material that is not required for docking is removed with the help of Autodock. It is a software used for molecular modelling. Many scientists and researchersuse this software to study the proteinligand interaction. This software is freely accessible under GNU's Not Unix (GNU) General Public License. It is the most favourable docking software used and cited by many researchers and scientists(W. Forli et. al. (2012).

Patchdock and Firedock: PDB format files of ligand and protein were uploaded to patch dock and 3D docked models were accessed after that firedock was performed with the 3D docked models. These are online free and easily accessible server that are used for the ligand - protein docking. They 
run their own algorithm to perform the docking. Results are obtained in a type of link on the personal email given in the server. By clicking on that link we can access the different docked models of Ligand - protein.

Figure 7: 2D Docked Structure Phloroglucinol and $M^{\text {pro }}$

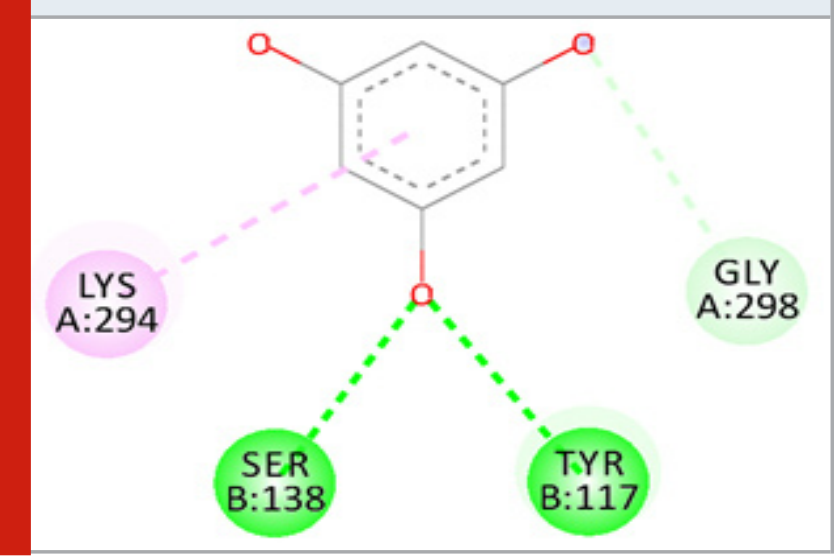

Drug Discovery Studio: 3D docked model files was downloaded and visualised in BOVIA Drug Discovery Studio. This software is useful for many scientists as they have to mind many parameters like they have to optimize biochemical potency and ADME and toxicity, then this software helps them to overcome this problem. It also helps in showing the protein-ligand interaction.

Table 1. Table representing the Bonds type and distance between the Ligand - Protein

\begin{tabular}{|l|c|c|c|}
\hline S.No. & Ligand - Protein & Distance & Bond type \\
\hline 1. & UNK0 - LYS294 & 4.76127 & Hydrophobic \\
\hline 2. & UNK0:O - TYR117 & 2.55842 & Hydrogen Bond \\
\hline 3. & UNK0 - SER138 & 2.42315 & Hydrogen Bond \\
\hline 4. & UNK0-GLY298 & 3.235 & Hydrogen Bond \\
\hline
\end{tabular}

Data Collection: Docked model with the highest Global energy was selcted as Global Energy or Gibbs free energy $(\Delta \mathrm{G})$ is same as the binding energy. It was recorded that Phloroglucinol was forming four bonds with the protein with the distance ranging from 2.4 to 4.7 (Table 1). One hydrophobic bonds was formed with LYS294 with the distance of 4.7 and three hydrogen bonds was formed with TYR117, SER138 and GLY298 with the distance of 2.5, 2.4 and 3.2 respectively as shown in Figure 6 and Figure 7.

\section{RESULT AND DISCUSSION}

After performing the docking, it was found that the docked model had an atomic contact energy (ACE) of $-90.41 \mathrm{kcal} /$ $\mathrm{mol}$, this is the energy of replacing the protein-atom contact with protein atom/water contactand a Global energy of -19.32. Negative value of $\Delta \mathrm{G}$ depicts that protein-ligand binding is achieved. Since the binding of protein-ligand is calculated by the scale of negative $\Delta \mathrm{G}$, it can be said that $\Delta \mathrm{G}$ determines the stability of protein-ligand complex or the binding energy of a ligand to the receptor.Docked three dimensional and two dimensional models also revealed that ligand is interacting with the protein with less distance between them. Current research suggests that Phloroglucinolcan be a potential inhibitor of Main protease

\section{CONCLUSION}

In 2109, Corona Virus emerged as a pandemic and many countries faced loss of life and financial crisis. Many countries are still facing the disease and millions of people are dying due to unavailability of a proper drug or vaccine against this disease. Many natural compounds are being used to treat viral diseases. This research was conducted to present that the phloroglucinol from brown algae acts as a promising drug to stop and cure corona virus. Phloroglucinol showed excellent drug likeliness with good parameters. Finally this research proves that the phloroglucinol can be promising anti-viral against SARS CoV2 Main Proteaseshowing a good value Of ACE and Global energy and the distance between the bonds. Further in-vitro studies and in-vivo studies are needed in this topic to develop a proper vaccine or drug for the treatment of coronavirus.

\section{REFERENCES}

COVID, W., 19. Coronavirus Pandemic: Coronavirus Cases.

Chojnacka, K., Witek-Krowiak, A., Skrzypczak, D., Mikula, K. and Młynarz, P., 2020. Phytochemicals containing biologically active polyphenols as an effective agent against Covid-19-inducing coronavirus. Journal of Functional Foods, p.104146.

Ezhilarasi, G.D., Latchoumi, T.P. and Balamurugan, K., 2020. UIP_A Smart Web Application to Manage Network Environments, Advances in Intelligent systems and computing book series.

Goswami, S.K., Gangadarappa, S.K., Vishwanath, M., Razdan, R., Jamwal, R., Bhadri, N. and Inamdar, M.N., 2016. Antioxidant potential and ability of phloroglucinol to decrease formation of advanced glycation end products increase efficacy of sildenafil in diabetes-induced sexual dysfunction of rats. Sexual medicine, 4(2), pp.e106e114.

Kandeel, M. and Al-Nazawi, M., 2020. Virtual screening and repurposing of FDA approved drugs against COVID19 main protease. Life sciences, 251, p.117627.

Kim, Y.C., An, R.B., Yoon, N.Y., Nam, T.J. and Choi, J.S., 2005. Hepatoprotective constituents of the edible brown alga Ecklonia stolonifera on tacrine-induced cytotoxicity in Hep G2 cells. Archives of pharmacal research, 28(12), pp.1376-1380.

Mishra, P., Jimmy, L., Ogunmola, G.A., Phu, T.V., Jayanthiladevi, A. and Latchoumi, T.P., 2020, December. Hydroponics cultivation using real time iot measurement 
system. In Journal of Physics: Conference Series (Vol. 1712, No. 1, p. 012040). IOP Publishing.

Prasath, S. (2020). Probabilistic Mceliece Public-Key Cryptography Based Identity Authentication For Secured Communication In Vanet. Solid State Technology, 63(6), 10167-10182.

Prasath, S. Validating Data Integrity in Steganographed Images using Embedded Checksum Technique. International Journal of Computer Applications, 975, 8887.

Ryu, J., Zhang, R., Hong, B.H., Yang, E.J., Kang, K.A., Choi, M., Kim, K.C., Noh, S.J., Kim, H.S., Lee, N.H. and Hyun, J.W., 2013. Phloroglucinol attenuates motor functional deficits in an animal model of Parkinson's disease by enhancing Nrf2 activity. PloS one, 8(8), p.e71178.

SAMANT, L. and Javle, V., 2020. Comparative docking analysis of rational drugs against COVID-19 main protease.

Venkata Pavan, M., Karnan, B. and Latchoumi, T.P., 2021. PLA-Cu reinforced composite filament: Preparation and flexural property printed at different machining conditions. Advanced Composite Materials, https://doi. org/10.1080/09243046.2021, 1918608.

Wang, B., Wang, A., Cao, Z. and Zhu, G., 2016. Characterization of a novel highly thermostable esterase from the Gram-positive soil bacterium Streptomyces lividans TK64. Biotechnology and applied biochemistry, 63(3), pp.334-343.

Yang, E.J., Ahn, S., Ryu, J., Choi, M.S., Choi, S., Chong, Y.H., Hyun, J.W., Chang, M.J. and Kim, H.S., 2015. Phloroglucinol attenuates the cognitive deficits of the 5XFAD mouse model of Alzheimer's disease. PLoS One, 10(8), p.e0135686. 